\title{
Concerns and psychosocial reactions to cancer recurrence were different from those occurring at the initial diagnosis
}

\author{
Mahon SM, Casperson DM. Exploring the psychosocial meaning of recurrent cancer: a descriptive study. Cancer Nurs 1997 Jun; \\ 20:178-86.
}

\section{Objective}

To describe the meaning of cancer recurrence to the patient and to explore if the patient perceives the diagnosis of recurrence as being different from the initial diagnosis of cancer.

\section{Design}

In depth interviews based on the conceptual framework of Lazarus and Folkman's model of stress, appraisal, and coping.

\section{Setting}

A large midwestern city in the USA.

\section{Patients}

20 patients $>21$ years of age (mean age 54 y, range $26-72,65 \%$ women) with a recurrence of cancer diagnosed within the previous 30 days who were having antitumour treatment. Most patients had lived for $>4$ years (mean 50 months) with a diagnosis of cancer. Inclusion criteria were the ability to speak, read, and write English; life expectancy $\geqslant 6$ months; and at home for $\geqslant 2$ weeks after the diagnosis.

\section{Methods}

Each subject participated in an interview that was partly semistructured and partly unstructured, averaging 67 minutes. Interviews were audiotaped, transcribed verbatim, and coded concurrently. No new themes emerged after the 9th interview, suggesting data saturation.

\section{Main results}

5 themes emerged concerning how the patient interpreted the recurrence. Firstly, most patients were able to describe some specific distress or a landmark event that they felt had led to the recurrence.
Secondly, related to the diagnosis itself, many patients had some symptoms before the diagnosis that they initially attributed to some other cause. While many knew that recurrence was possible, all stated that they could never have been prepared for the shock of being told of the recurrence.

Thirdly, previous experiences with cancer affected the way the patients perceived the recurrence. Patients with previous recurrences were not surprised by the diagnosis and were hopeful of a remission. Patients with negative past experiences with recurrence in family or friends viewed their prognosis as poor.

Fourthly, all patients discussed issues related to death and dying and for many, the recurrence triggered a fear that they would die prematurely.

Fifthly, all patients were able to identify some positive aspects as a result of the recurrence, such as a stronger appreciation for life, and a shift in focus from career advancement to family and life long goals.

3 themes emerged that were related to differences between the initial cancer diagnosis and the recurrence: a deeper awareness of the significance of the diagnosis; more difficulty with treatment because of their fears, past memories, and more time to worry and dread the treatment; and an awareness of health care system changes (decreased length of stay and increased outpatient service use).

\section{Conclusion}

The recurrence of cancer led patients to experience a wide array of concerns and psychosocial problems that were distinct from the initial diagnosis.

Source of funding: National Institutes of Health.

For article reprint:Dr S M Mahon, 9322 Hazelridge Drive, St.Louis, MO 63126, USA. Fax +1314 7685620.

\section{Commentary}

Although cancer recurrence is well documented in the literature, this study by Mahon and Casperson is unique for 2 reasons. Firstly, the study explores the significance of a recent diagnosis of recurrence of cancer and its impact on patients. Secondly, the study uses a stress appraisal and coping framework. Most often stress coping studies report the end points or degrees of change but do little to illuminate the process. This study describes the feelings aroused by recurrence and reports the attributions that influenced the thinking of the patients, explained the recurrence, and accounted for the sense of hope or hopelessness.
These findings add substantially to nursing knowledge, reminding us to ask, assess, and inform relative to the thoughts and attributions of individual patients at that time.

Strengths of the study include pilot testing and revision of the interview guide based on individual responses, as well as clarity of operational definitions and rationale for sample selection. Coding was developed, tested using 2 coders, and a summary returned to 3 people for validation. Findings are clearly presented but lack richness of description, which may be attributed to the single interview per person format.
The findings of the study suggest that nurses may have a major part to play in the support, education, and follow up of new recurrences in patients with cancer. The findings provide a context for working with patients with recurrent cancer. Nurses may need to be direct in asking patients about specific concerns, thoughts, and feelings surrounding the recurrence to provide appropriate education and ongoing psychosocial support.

Dauna Crooks, RN, DNS Research Associate Supportive Cancer Care Research Unit Hamilton, Ontario, Canada 